\title{
Dispersão e comportamento reológico de concretos refratários ultra-baixo teor de cimento no sistema $\mathrm{Al}_{2} \mathrm{O}_{3}-\mathrm{SiC}_{-} \mathrm{SiO}_{2}-\mathrm{C}$ (Dispersion and setting control of ultra-low cement refractory castables in the $\mathrm{Al}_{2} \mathrm{O}_{3}-\mathrm{SiC}-\mathrm{SiO}_{2}-\mathrm{C}$ system)
}

\author{
I. R. de Oliveira ${ }^{1}$, A. R. Studart ${ }^{1}$, B. A. Menegazzo ${ }^{2}$, V. C. Pandolfelli ${ }^{1}$ \\ ${ }^{1}$ Universidade Federal de S. Carlos, DEMa \\ Rod. Washington Luiz, km 235, C.P. 676, S. Carlos, SP, 13565-905 \\ ${ }^{2}$ Alcoa Aluminio S.A. \\ ivone@iris.ufscar.br,vicpando@power.ufscar.br
}

\begin{abstract}
Resumo
Concretos refratários no sistema $\mathrm{Al}_{2} \mathrm{O}_{3}-\mathrm{SiC}-\mathrm{SiO}_{2}-\mathrm{C}$ vêm sendo amplamente utilizados em indústrias siderúrgicas como revestimento de canais de corrida de altos-fornos, em virtude principalmente da sua elevada refratariedade aliada a altas resistências ao choque térmico e ao ataque por escória e metal fundido. Neste trabalho, investigou-se a influência de diferentes tipos de aditivos na trabalhabilidade e dispersão de concretos refratários de ultra-baixo teor de cimento nesse sistema. Apesar da sua alta capacidade de complexar íons de cálcio, moléculas de citrato não foram capazes de controlar adequadamente o tempo de pega do concreto estudado, contradizendo a idéia geral de que os íons de citrato devem ser utilizados para controlar a sua trabalhabilidade. Por outro lado, o aditivo polimetacrilato de sódio mostrou-se eficiente na otimização simultânea da dispersão e da trabalhabilidade do concreto devido provavelmente ao retardamento da dissolução dos íons advindos do cimento.

Palavras-chave: concreto, reologia, trabalhabilidade, dispersão, refratários.
\end{abstract}

Abstract

Refractory castables in the $\mathrm{Al}_{2} \mathrm{O}_{3}-\mathrm{SiC}-\mathrm{SiO}{ }_{2}$ - C system have been extensively used as linings for blast furnace runners, due mainly to their improved resistance to thermal shock damage and to slag and metal corrosion, respectively. In this work, ULC refractory castables in the $\mathrm{Al}_{2} \mathrm{O}_{3}-\mathrm{SiC}-\mathrm{SiO}_{2}-\mathrm{C}$ system were prepared in order to evaluate the efficiency of different sort of additives on their dispersion and setting behavior. Although citrate ions are known to be efficient chelating agents, they were not able to properly control the working time of the castable studied, contradicting the general idea that citrate ions are necessary for controlling castable setting. On the other hand, the sodium polymethacrylate additive was found to be more efficient for the simultaneous optimization of the castable dispersion state and working time. This may be attributed to a retardation effect imparted by polymethacrylate molecules on the dissolution of ions from the surface of cement particles.

Keywords: castable, rheology, setting, dispersion, refractories.

\section{INTRODUÇÃO}

O caráter empírico dos estudos envolvendo a dispersão de concretos refratários tem estimulado uma avaliação sistemática da atuação de dispersantes sobre a fluidez e o comportamento reológico de concretos refratários de alta alumina.

Como resultado, diversos estudos têm sido efetuados para investigar a dispersão de concretos zero-cimento, abrangendo desde sistemas bastante simples contendo apenas $\mathrm{Al}_{2} \mathrm{O}_{3}[1]$, até os mais complexos e próximos aos concretos comerciais como, por exemplo, concretos no sistema $\mathrm{Al}_{2} \mathrm{O}_{3}$-SiC-C [2]. Nesses estudos, nenhum tipo de ligante hidráulico ou cimento foi utilizado visando simplificar a avaliação da atuação de diferentes tipos de aditivos sobre a dispersão e o comportamento reológico dos concretos.

A adição de cimento constitui uma dificuldade adicional ao estudo da dispersão devido à característica mutante dessa matéria-prima, que provoca a elevação da força iônica do meio em função do tempo e a precipitação de fases hidratadas. Essa complexidade dos concretos contendo cimento associado à inferior refratariedade desses materiais [3], motivaram o estudo da dispersão em sistemas zero-cimento $[1,2]$.

Entretanto, o cimento ainda é um agente coagulante largamente utilizado em aplicações industriais devido à sua grande disponibilidade, baixo custo e capacidade de conferir alta resistência mecânica aos concretos a temperatura ambiente. Em virtude disso, o principal objetivo desse trabalho é investigar a influência de diferentes tipos de aditivos na trabalhabilidade e dispersão de concretos refratários contendo cimento. Essa avaliação foi efetuada em concretos de ultrabaixo teor de cimento no sistema $\mathrm{Al}_{2} \mathrm{O}_{3}-\mathrm{SiC}-\mathrm{SiO}_{2}-\mathrm{C}$ normalmente utilizados no revestimento de canais de corrida de altos-fornos. A associação dessas matérias-primas tem elevado o desempenho desses materiais frente às severas condições de trabalho, devido ao aumento da resistência ao choque térmico e ao ataque por escória e metal fundido. 


\section{MATERIAIS E MÉTODOS}

Concretos refratários no sistema $\mathrm{Al}_{2} \mathrm{O}_{3}-\mathrm{SiC}-\mathrm{SiO}_{2}-\mathrm{C}$ foram preparados com uma composição similar à uma composição comercial de concreto refratário utilizado como revestimento para canais de corrida de altos-fornos, como apresentado na Tabela I.

Em virtude da presença de carbono na composição estudada, foi adicionado ao concreto o agente surfactante polyoxyethylene $(\mathrm{n}=20)$ cetyl ether (Brij 58, Sigma Aldrich Química, $1120 \mathrm{~g} / \mathrm{mol}$ ) a fim de promover a molhabilidade das partículas de coque e possibilitar a homogeneização da suspensão. Devido a importância desse aditivo, foi avaliada inicialmente a influência do seu teor sobre a dispersão e homogeneidade do concreto.

A preparação do concreto para a avaliação da influência do surfactante envolveu a pesagem de composições de $4 \mathrm{~kg}$ de acordo com a Tabela I, seguida da adição do aditivo defloculante (Darvan-7S) na forma sólida e a homogeneização em um misturador duplo cone por 5 minutos. Posteriormente, as composições foram misturadas utilizando-se um reômetro especial para concretos [4], seguindo um procedimento ótimo de mistura [5]. O agente surfactante foi dissolvido na água de mistura antes da preparação do concreto.

Após a mistura, os concretos foram submetidos a ciclos sucessivos de rotação (rotação variando entre 2 e $75 \mathrm{rpm}$ ) em função do tempo, sendo o intervalo entre os ciclos de 15 minutos. Após a mistura e cada ciclo foi efetuada a medida de fluidez usando um teste de fluidez livre ("free-flow") adaptado da norma ASTM C-860.

Em seguida avaliou-se a influência dos agentes defloculantes ácido cítrico (Labsynth; $P M=192 \mathrm{~g} / \mathrm{mol}$ ), citrato de sódio (Mercocítrico; 257,9 g/mol) e polimetacrilato de sódio (Darvan-7S; Vanderbilt; $2500 \mathrm{~g} / \mathrm{mol}$ ). Para complementar o estudo de dispersão, avaliou-se o efeito do teor de polimetacrilato de sódio nas propriedades reológicas do concreto estudado. $\mathrm{O}$ teor dos aditivos é expresso como a razão (massa do aditivo)/(área superficial do concreto).

A preparação do concreto para a avaliação da influência dos agentes defloculantes foi da mesma forma que a apresentada para a avaliação do surfactante, variando-se o tipo ou teor de defloculante adicionado, mantendo-se fixo o teor de surfactante. Neste caso, o concreto também foi submetido a uma rotação constante $(28 \mathrm{rpm})$ por um período definido pelo seu tempo de pega.

Os ensaios reológicos possibilitaram avaliar o comportamento do concreto quando submetido a altas taxas de cisalhamento, e a mudança de fluidez no decorrer do processo de pega hidráulica ou endurecimento. As variações de $\mathrm{pH}$ do concreto também foram acompanhadas em cada composição.

\section{RESULTADOS E DISCUSSÃO}

Espécies surfactantes possibilitam um aumento da molhabilidade de partículas de materiais hidrofóbicos (como o coque) pela água. Isso é possível, pelo fato desses aditivos associarem em uma mesma molécula grupos funcionais que apresentam elevada afinidade pelo meio líquido e grupos compatíveis com a superfície do sólido. Essas características possibilitam reduzir a energia interfacial entre sólidos hidrofóbicos e a água, atenuando a tendência de aglomeração desses materiais em meio aquoso [6].

O surfactante utilizado mostrou-se bastante eficiente, uma vez que possibilitou a obtenção de uma mistura homogênea sem a ocorrência de segregação das partículas de coque.

A Fig. 1 mostra que a presença de surfactante resultou em superiores valores de fluidez do concreto e menores níveis de torque durante os ciclos de cisalhamento os quais apresentaram valores de áreas de histerese muito próximos. O teor de $0,2 \mathrm{mg} / \mathrm{m}^{2}$ de surfactante foi determinado como sendo uma concentração ótima para a homogeneização e dispersão das partículas de coque.

Estudos anteriores mostraram que o ácido cítrico apresenta

Tabela I - Teores de matérias-primas e água usados para a preparação de concretos refratários no sistema $\mathrm{Al}_{2} \mathrm{O}_{3}-\mathrm{SiC}_{\mathrm{C}} \mathrm{SiO}_{2}-\mathrm{C}$. [Table I - Content of raw materials and water used to prepare the refractory castables in the $\mathrm{Al}_{2} \mathrm{O}_{3}$-SiC-SiO $\mathrm{O}_{2}$-C system.]

\begin{tabular}{|c|c|c|c|}
\hline & Matérias-primas & $\%$-volume & $\%$-peso \\
\hline \multirow[t]{2}{*}{ Agregados } & Alumina eletrofundida branca (EK8R, malha 5/16-200) & 51,1 & 57,0 \\
\hline & SiC (EC6R, malha 6-200) & 22,3 & 20,0 \\
\hline \multirow{5}{*}{ Finos } & $\mathrm{Al}_{2} \mathrm{O}_{3}$ calcinada A-3000FL & 9,0 & 10,0 \\
\hline & $\mathrm{Al}_{2} \mathrm{O}_{3}$ calcinada $\mathrm{A}-2 \mathrm{G}$ & 2,7 & 3,0 \\
\hline & Coque (Unicarbo 50) & 3,6 & 2,0 \\
\hline & Microssílica (971-D) & 7,9 & 5,0 \\
\hline & Cimento (CA270) & 3,4 & 3,0 \\
\hline água destilada & & 15,1 & 5,0 \\
\hline
\end{tabular}

Área superficial do concreto $=1,54 \mathrm{~m}^{2} / \mathrm{g}$

Fornecedor das matérias-primas: Alcoa Alumínio, exceto coque (Unimetal) e microssílica (Elkem Refractories). 

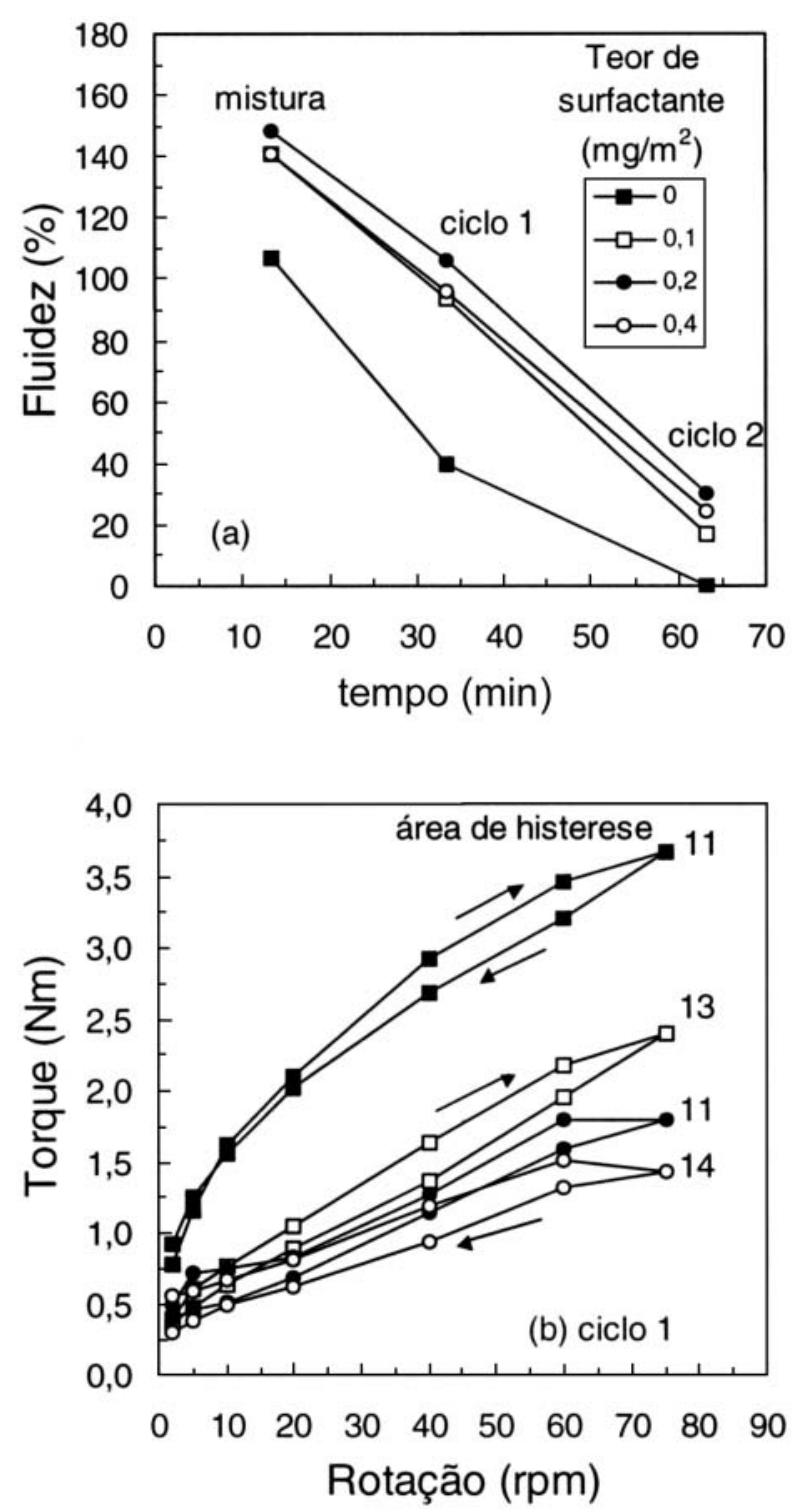

Figura 1: Curvas de fluidez em função do tempo (a) e de torque em função da rotação (b) para composições de concreto $\mathrm{Al}_{2} \mathrm{O}_{3}-\mathrm{SiC}_{-} \mathrm{SiO}_{2}$ $\mathrm{C}$ (5\%-peso de água) na presença de $0,97 \mathrm{mg} / \mathrm{m}^{2}$ de polimetacrilato de sódio $(2500 \mathrm{~g} / \mathrm{mol})$ e diferentes teores de surfactante.

[Figure 1: (a) Free-flow as a function of the time and (b) torque as a function of the rotation of $\mathrm{Al}_{2} \mathrm{O}_{3}-\mathrm{SiC}-\mathrm{SiO}-\mathrm{C}$ castables compositions containing $5 \mathrm{wt} \%$ of water, in the presence of $0.97 \mathrm{mg} / \mathrm{m}^{2}$ of sodium polymethacrylate $(2500 \mathrm{~g} / \mathrm{mol})$ and different surfactant contents.]

bastante eficiência na dispersão do sistema $\mathrm{Al}_{2} \mathrm{O}_{3}-\mathrm{SiC}-\mathrm{C}$ [2]. Em virtude disso, foi inicialmente avaliada a eficiência desse aditivo na dispersão do sistema $\mathrm{Al}_{2} \mathrm{O}_{3}-\mathrm{SiC}_{2} \mathrm{SiO}_{2}-\mathrm{C}$ contendo cimento utilizando-se um teor de $0,4 \mathrm{mg} / \mathrm{m}^{2}$, o qual corresponde a um teor ótimo para a dispersão de concretos zero-cimento no sistema $\mathrm{Al}_{2} \mathrm{O}_{3}$-SiC-C [2]. Entretanto, a Fig. 2 mostra que para o atual sistema esse teor proporciona um tempo de pega hidráulica muito curto ( $~ 30$ minutos).

Para compreender o efeito dos aditivos sobre a trabalhabilidade dos concretos UBTC é necessário analisar o mecanismo de pega hidráulica promovido pelo cimento. A pega hidráulica é um fenômeno de dissolução de íons e subsequente precipitação de hidratos. Quando ocorre a mistura do cimento em água, há a dissociação dos compostos iniciais (fases anidras), gerando uma solução com íons de cálcio $\left(\mathrm{Ca}^{2+}\right)$ e hidróxido de alumínio $\left(\mathrm{Al}(\mathrm{OH})_{4}^{-}\right)$. Quando a solução torna-se saturada, esses íons começam a nuclear e precipitar na forma de hidratos de aluminato de cálcio, tornando a solução novamente insaturada e, portanto capaz de dissolver outras fases anidras. O processo então prossegue com a nucleação e crescimento de cristais, que vão precipitando e formando uma estrutura rígida entre as partículas [7].

Um dos mecanismos pelos quais um aditivo pode funcionar como um retardador da pega hidráulica é através da formação de compostos insolúveis com os íons $\mathrm{Ca}^{2+}$ presentes na solução, fazendo com que seja necessária a dissociação de uma maior quantidade da fase anidra para que ocorra a precipitação de hidratos.

Tendo em vista o conhecido efeito "complexante" dos íons de citrato sobre os íons de cálcio, teores superiores de ácido cítrico foram testados visando aumentar a trabalhabilidade do concreto. Os resultados obtidos (Fig. 2) mostraram que o aumento do teor de ácido cítrico possibilita, de fato, um aumento do tempo de pega do concreto. No entanto, verificouse que mesmo com um teor bastante elevado de ácido cítrico, o concreto apresentou baixa trabalhabilidade quando o teor de água da composição foi reduzido para um valor

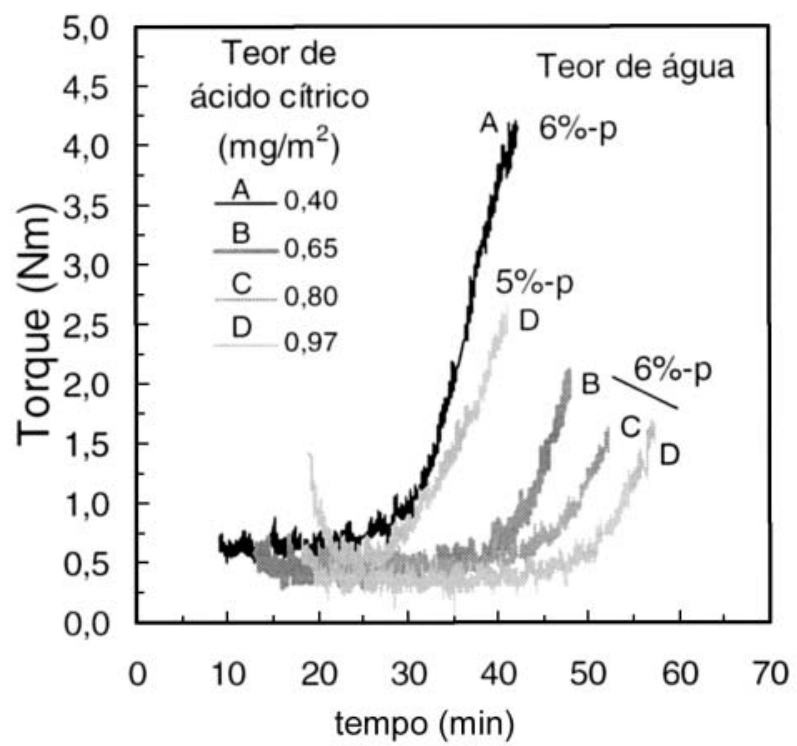

Figura 2: Curvas de torque em função do tempo mantendo-se rotação constante $(28 \mathrm{rpm})$ para composições de concreto $\mathrm{Al}_{2} \mathrm{O}_{3}-\mathrm{SiC}-\mathrm{SiO}_{2}-\mathrm{C}$ na presença de $0,2 \mathrm{mg} / \mathrm{m}^{2}$ de surfactante contendo diferentes teores de ácido cítrico (A, B, C, D) e com teores de água de 5 e 6\%-peso. [Figure 2: Torque as a function of the time (28 rpm) for $\mathrm{Al}_{2} \mathrm{O}_{3}-\mathrm{SiC}$ $\mathrm{SiO}_{2}-\mathrm{C}$ castables compositions with 5 and $6 \mathrm{wt} \%$ of water, in the presence of $0.20 \mathrm{mg} / \mathrm{m}^{2}$ of surfactant and different citric acid contents (A, B, C, D).] 

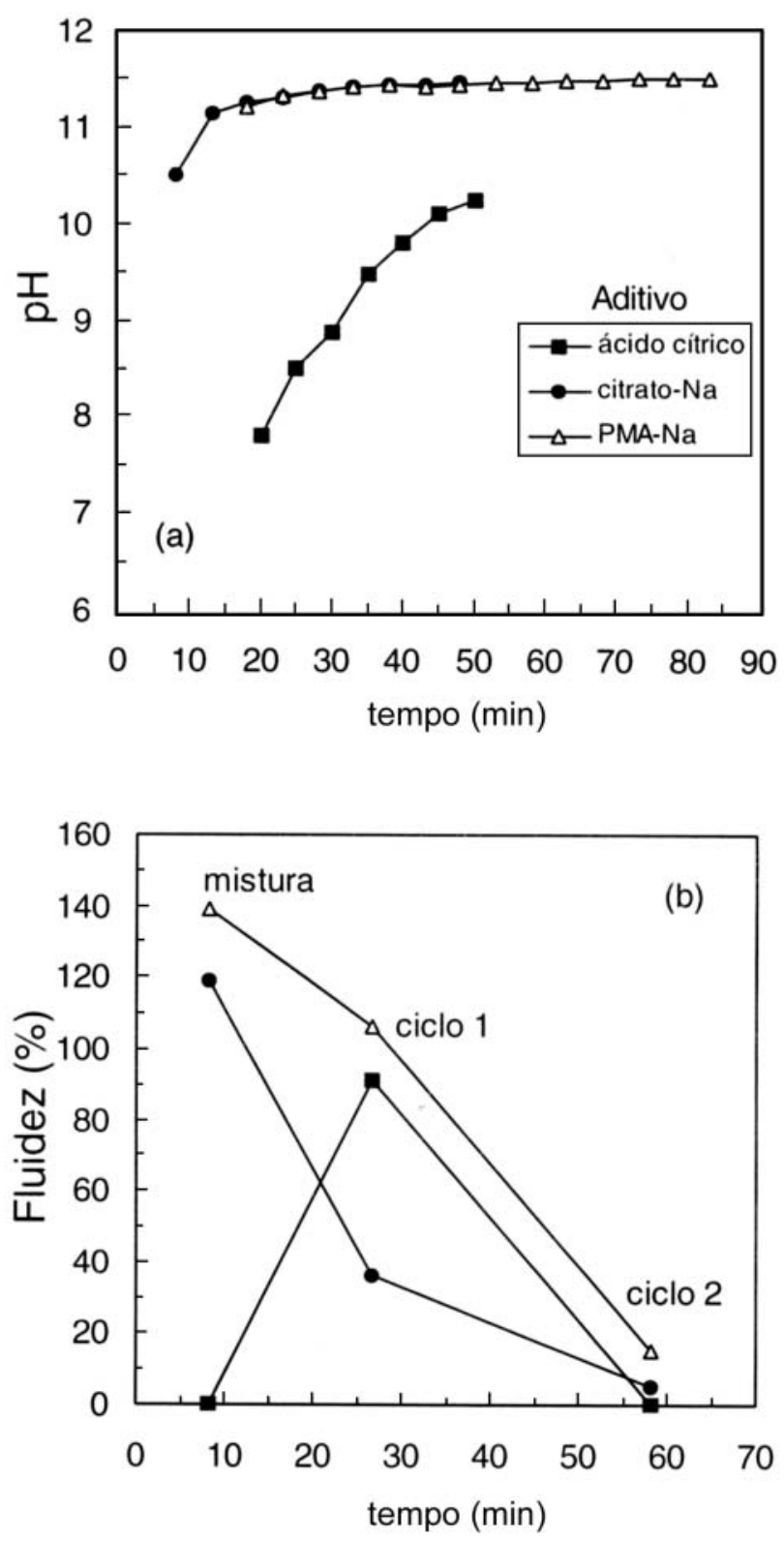

Figura 3: Variação do pH (a) e da fluidez (b) em função do tempo para composições de concreto $\mathrm{Al}_{2} \mathrm{O}_{3}-\mathrm{SiC}_{-} \mathrm{SiO}_{2}-\mathrm{C}$ (5\%-peso de água) na presença de $0,2 \mathrm{mg} / \mathrm{m}^{2}$ de surfactante e $0,97 \mathrm{mg} / \mathrm{m}^{2}$ de ácido cítrico, citrato de sódio ou polimetacrilato de sódio.

[Figure 3: Variation of (a) $\mathrm{pH}$ and (b) free-flow as a function of time for $\mathrm{Al}_{2} \mathrm{O}_{3}-\mathrm{SiC}-\mathrm{SiO}_{2}-\mathrm{C}$ castables compositions containing $5 \mathrm{wt} \%$ of water, in the presence of $0.20 \mathrm{mg} / \mathrm{m}^{2}$ of surfactant and $0.97 \mathrm{mg} / \mathrm{m}^{2}$ of different additives: citric acid, sodium citrate and sodium polymethacrylate.]

normalmente utilizado em aplicações industriais (5\%-p).

Além disso, a adição de um elevado teor de ácido cítrico ao concreto provocou uma queda acentuada do $\mathrm{pH}$, reduzindo a fluidez inicial da composição (Fig. 3). Um aumento de fluidez foi verificado somente cerca de 30 minutos após a mistura, o que pode ser atribuído ao aumento do $\mathrm{pH}$ do concreto para uma faixa de valores ótima de dispersão.

$\mathrm{O}$ problema de queda acentuada do $\mathrm{pH}$ do concreto foi

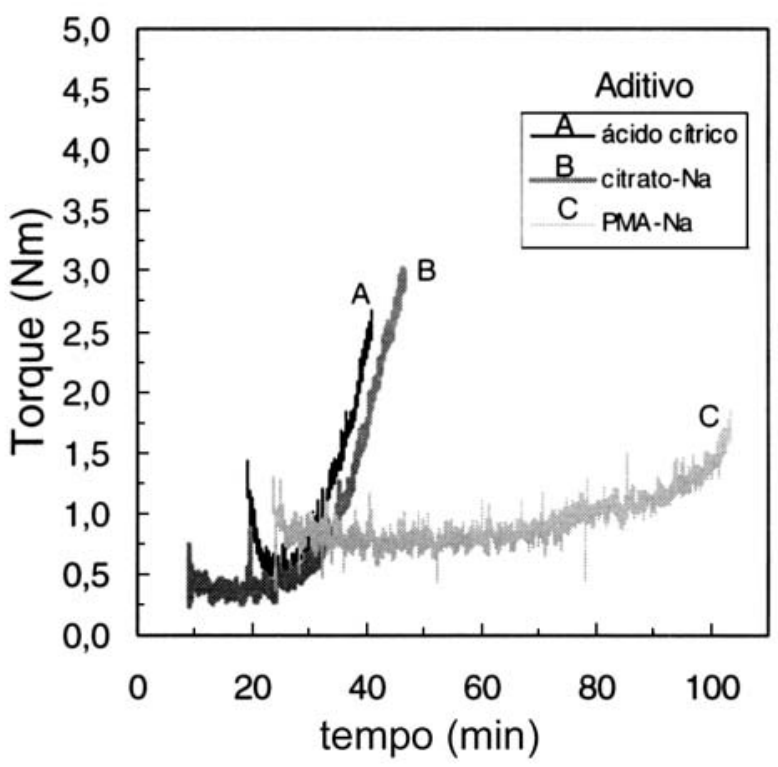

H1gura 4: (urvas de torque em tunçao do tempo mantendo-se rotaçao constante $(28 \mathrm{rpm})$ para composições de concreto $\mathrm{Al}_{2} \mathrm{O}_{3}-\mathrm{SiC}-\mathrm{SiO}_{2}-\mathrm{C}$ ( $5 \%$-peso de água) na presença de $0,2 \mathrm{mg} / \mathrm{m}^{2}$ de surfactante e 0,97 $\mathrm{mg} / \mathrm{m}^{2}$ de ácido cítrico (A), citrato de sódio (B) ou polimetacrilato de sódio (C).

[Figure 4: Torque as a function of time (28 rpm) of $\mathrm{Al}_{2} \mathrm{O}_{3}-\mathrm{SiC}-\mathrm{SiO}_{2}-\mathrm{C}$ castables compositions containing $5 \mathrm{wt} \%$ of water, in the presence of $0.20 \mathrm{mg} / \mathrm{m}^{2}$ of surfactant and $0.97 \mathrm{mg} / \mathrm{m}^{2}$ of different additives: citric acid (A), sodium citrate $(B)$ and sodium polymethacrylate $(C)$.]

contornado utilizando-se citrato de sódio como dispersante. Na presença desse aditivo, obteve-se um grande aumento de fluidez após a mistura. Entretanto, os concretos preparados com citrato de sódio ainda apresentaram baixa trabalhabilidade, como apresentado na Fig. 4.

Portanto, apesar da sua alta capacidade de complexar íons de cálcio, os íons de citrato não foram capazes de controlar adequadamente o tempo de pega dos concretos. Esse resultado, a princípio surpreendente, contradiz a idéia geral de que os íons de citrato devem ser utilizados para controlar a pega do cimento.

Por outro lado, os resultados apresentados mostraram a superior eficiência do polimetacrilato de sódio (Darvan-7S) na otimização da dispersão e trabalhabilidade do concreto estudado.

Na presença desse aditivo, foram obtidos os maiores valores de fluidez do concreto após a mistura e os ciclos de cisalhamento. As curvas de torque em função da rotação (ciclos de histerese) obtidas para o primeiro e segundo ciclos (Fig. 5), confirmam a dispersão eficiente do sistema na presença de polimetacrilato de sódio. Neste caso, observa-se os menores níveis de torque e valores de área da histerese. Menores níveis de torque de área de histerese estão associados a uma melhor dispersão das partículas, uma vez que o concreto adequadamente disperso oferece menor resistência ao cisalhamento, reduzindo o torque necessário para misturá-lo em uma determinada rotação.

É importante salientar que os ciclos de cisalhamento apresentados na Fig. 5 fornecem energia adicional à fornecida durante a mistura para a eventual quebra de aglomerados presentes. Num sistema bem disperso, esta energia adicional 

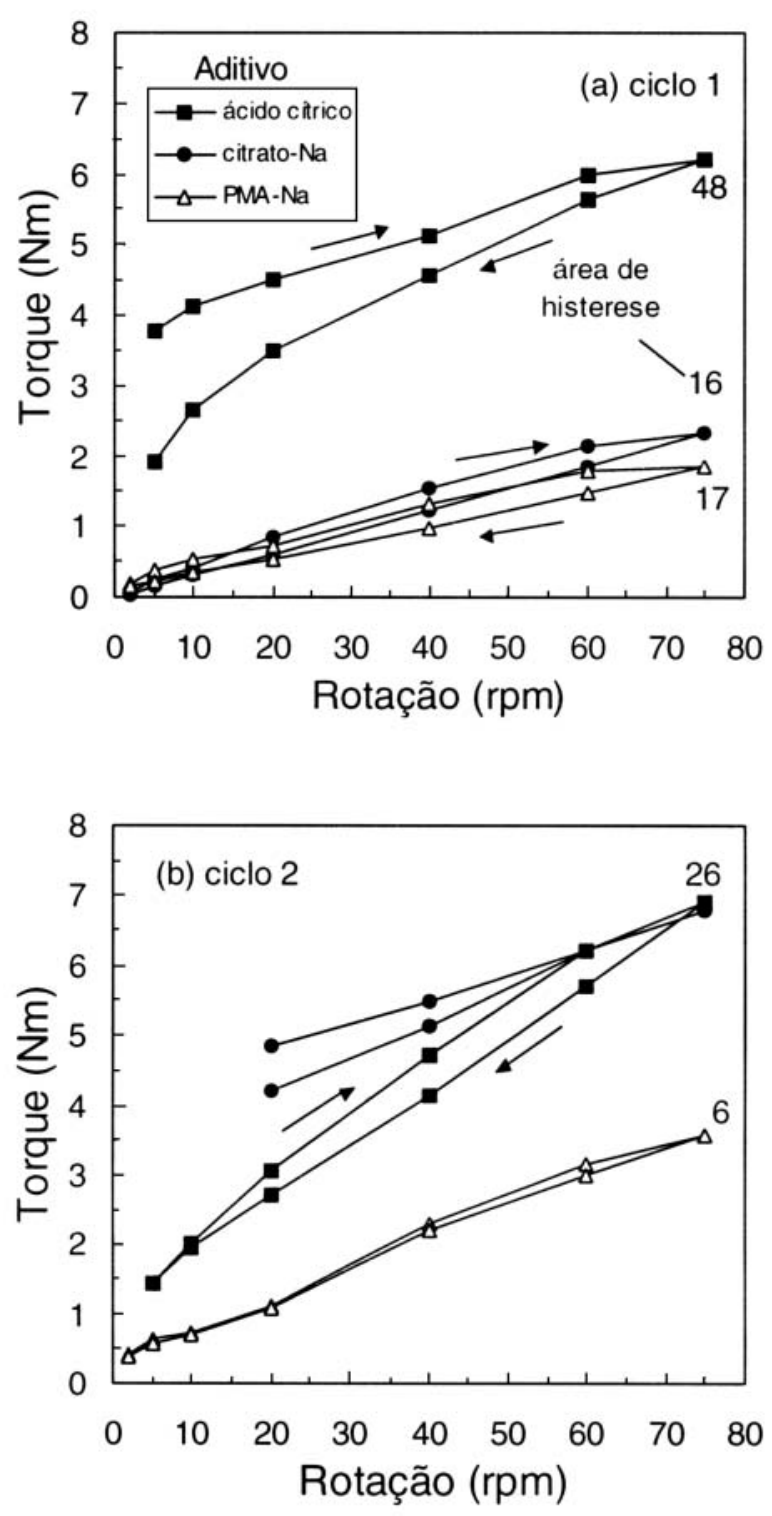

Figura 5: Curvas de torque em função da rotação para composições de concreto $\mathrm{Al}_{2} \mathrm{O}_{3}-\mathrm{SiC}-\mathrm{SiO}_{2}-\mathrm{C}$ (5\%-peso de água) na presença de 0,2 $\mathrm{mg} / \mathrm{m}^{2}$ de surfactante e $0,97 \mathrm{mg} / \mathrm{m}^{2}$ de ácido cítrico, citrato de sódio ou polimetacrilato de sódio obtidas para o primeiro (a) e segundo (b) ciclos.

[Figure 5: Torque as a function of the mixer revolution for the $\mathrm{Al}_{2} \mathrm{O}_{3}$ $\mathrm{SiC}-\mathrm{SiO}-\mathrm{C}$ castables compositions containing $5 \mathrm{wt} \%$ water, in the presence of $0.20 \mathrm{mg} / \mathrm{m}^{2}$ of surfactant and $\quad 0.97 \mathrm{mg} / \mathrm{m}^{2}$ of different additives: citric acid, sodium citrate and sodium polymethacrylate. The data were obtained for (a) first and (b) second shear cycles.]

promove o aquecimento do concreto, que somado aos efeitos da hidratação do cimento resultam na queda de fluidez. Por outro lado, em um sistema mal disperso, como é o caso da composição com ácido cítrico, a quebra de aglomerados durante o ciclo pode resultar em um aumento de fluidez. O elevado valor de área da histerese verificado durante o ciclo aplicado aos concretos contendo ácido cítrico pode ser um
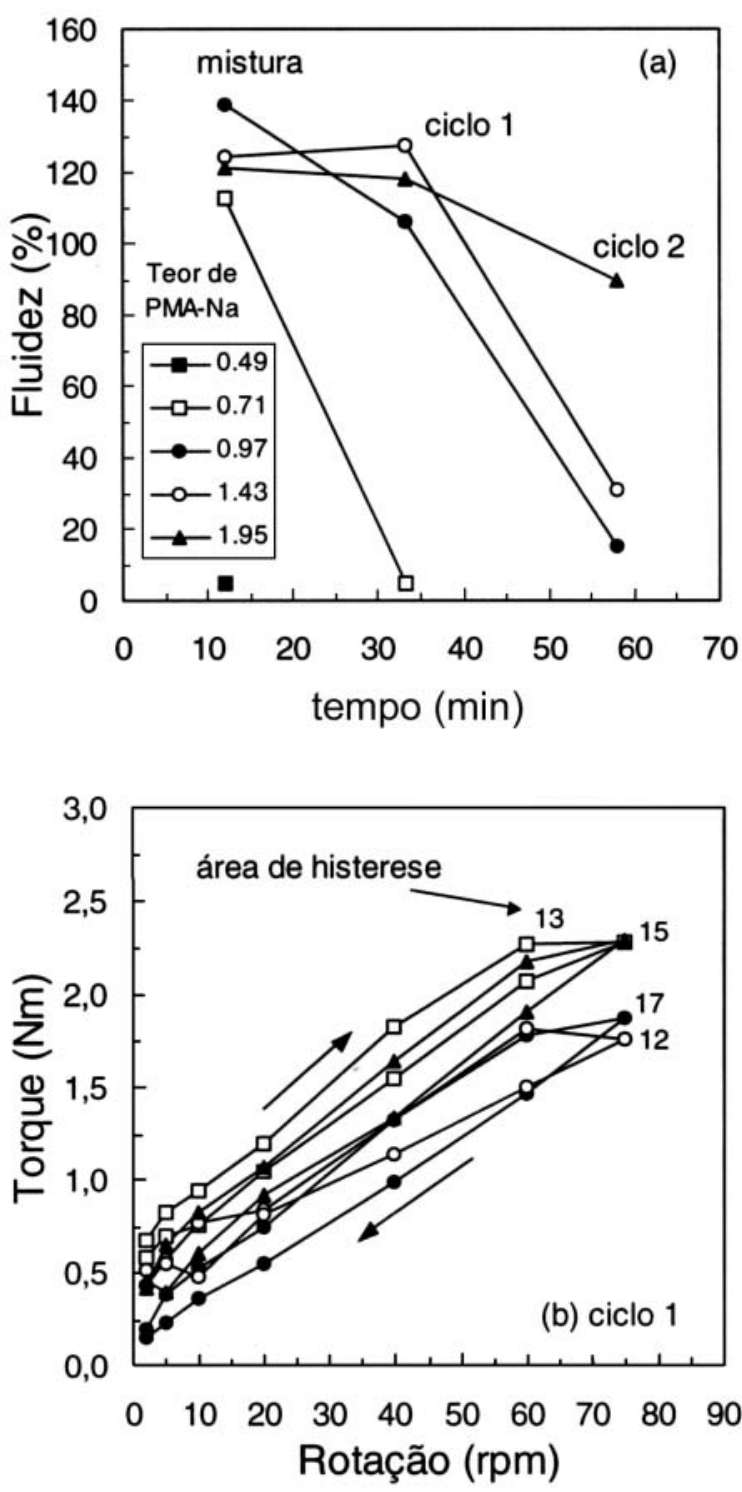

Figura 6: Curvas de fluidez em função do tempo (a) e de torque em função da rotação (b) para composições de concreto $\mathrm{Al}_{2} \mathrm{O}_{3}-\mathrm{SiC}-\mathrm{SiO}_{2}-$ $\mathrm{C}$ (5\%-peso de água) na presença de $0,2 \mathrm{mg} / \mathrm{m}^{2}$ de surfactante $\mathrm{e}$ diferentes teores $\left(\mathrm{mg} / \mathrm{m}^{2}\right)$ de polimetacrilato de sódio.

[Figure 6: (a) Free-flow curves as a function of time and (b) torque as a function of the mixer revolution for $\mathrm{Al}_{2} \mathrm{O}_{3}-\mathrm{SiC}-\mathrm{SiO}_{2}-\mathrm{C}$ castables compositions containing $5 \mathrm{wt} \%$ water, in the presence of $0.20 \mathrm{mg} / \mathrm{m}^{2}$ of surfactant and different content $\left(\mathrm{mg} / \mathrm{m}^{2}\right)$ of sodium polymethacrylate.]

indicativo de que parte da energia fornecida durante o ciclo foi empregada na quebra de aglomerados presentes nesses concretos. Esse efeito associado ao aumento de $\mathrm{pH}$ em função do tempo (Fig. 3) foram provavelmente os fatos responsáveis pelo aumento de fluidez observado nos concretos preparados com ácido cítrico.

Quanto ao efeito do polimetacrilato de sódio sobre a 


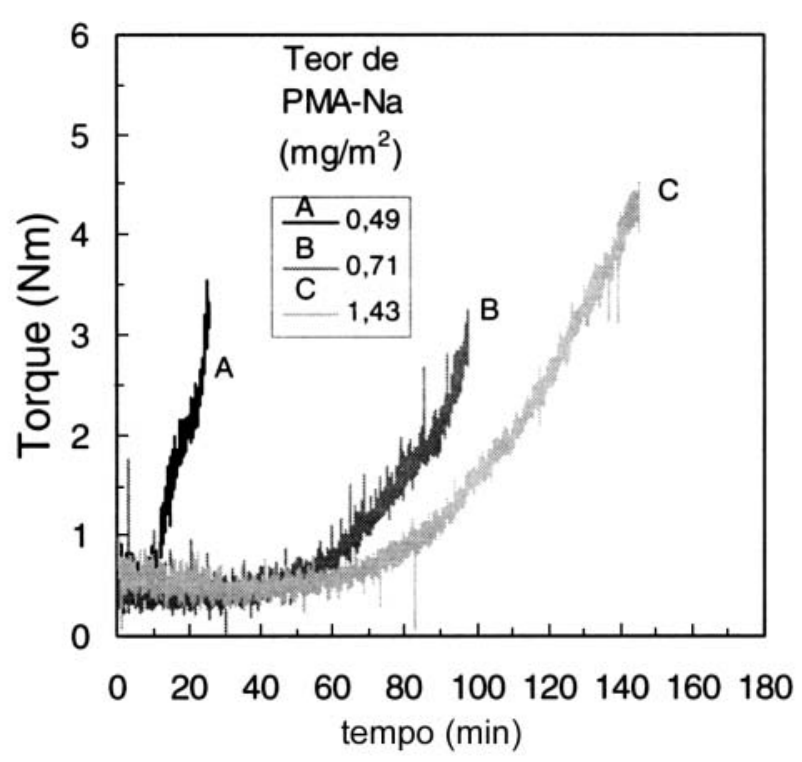

Figura 7: Curvas de torque em função do tempo mantendo-se rotação constante $(28 \mathrm{rpm})$ para composições de concreto $\mathrm{Al}_{2} \mathrm{O}_{3}-\mathrm{SiC}_{-} \mathrm{SiO}_{2}-\mathrm{C}$ com 5\%-p de água, na presença de $0,20 \mathrm{mg} / \mathrm{m}^{2}$ de surfactante e diferentes teores de polimetacrilato de sódio (A, B, C).

[Figure 7: Torque as a function of time (28 rpm) of $\mathrm{Al}_{2} \mathrm{O}_{3}-\mathrm{SiC}_{-} \mathrm{SiO}_{2}-\mathrm{C}$ castables compositions containing $5 \mathrm{wt} \%$ of water, in the presence of $0.20 \mathrm{mg} / \mathrm{m}^{2}$ of surfactant and different content of sodium polymethacrylate $(A, B, C)$.]

trabalhabilidade do concreto, têm-se observado [8] que esse aditivo provoca um retardamento da dissolução dos íons advindos do cimento. Esse efeito pode estar relacionado com uma complexação mais eficiente dos íons de cálcio pelas moléculas de polimetacrilato ou com um impedimento físico e eletrostático na superfície das partículas de cimento que iniba a dissolução dos íons responsáveis pela pega hidráulica. Outros ensaios estão sendo realizados visando elucidar a atuação do polimetacrilato de sódio e do citrato de sódio sobre a hidratação do cimento [8].

Em virtude dos resultados satisfatórios obtidos com o polimetacrilato de sódio, avaliou-se a influência do teor desse aditivo sobre a dispersão do concreto estudado.

A Fig. 6-a mostra que o teor de polimetacrilato de sódio afeta principalmente as curvas de fluidez em função do tempo. Maiores valores de fluidez após a mistura foram obtidos para o teor de $0,97 \mathrm{mg} / \mathrm{m}^{2}$ de polimetacrilato. A adição de teores superiores proporcionaram queda na fluidez após a mistura. Entretanto, observa-se que para esses teores, a fluidez se mantém alta mesmo após o segundo ciclo de cisalhamento. A princípio, não podemos correlacionar tais níveis de fluidez com a quebra de aglomerados promovida pelos ciclos de cisalhamento, uma vez que não se observou uma redução significativa da área da histerese para os maiores teores utilizados (Fig. 6b). Por outro lado, o aumento do teor de polimetacrilato de sódio contribuiu para o aumento do tempo de pega do concreto justificando os altos valores de fluidez observados (Fig. 7).

\section{CONCLUSÕES}

Apesar da sua alta capacidade de complexar íons de cálcio, os íons de citrato não foram capazes de controlar adequadamente o tempo de pega do concreto $\mathrm{Al}_{2} \mathrm{O}_{3}-\mathrm{SiC}_{-} \mathrm{SiO}_{2}$ $\mathrm{C}$ estudado, contradizendo a idéia geral de que os íons de citrato devem ser utilizados para controlar a pega do cimento.

O polimetacrilato de sódio de peso molecular $2500 \mathrm{~g} / \mathrm{mol}$ mostrou-se muito eficiente na otimização da dispersão e da trabalhabilidade do concreto devido provavelmente ao retardamento da dissolução dos íons advindos do cimento. Acredita-se que esse efeito retardador possa estar relacionado à complexação mais eficiente dos íons de cálcio pelo polimetacrilato ou por um impedimento físico e eletrostático proporcionado pelo polimetacrilato na superfície das partículas de cimento que dificulte a dissolução dos íons responsáveis pela pega hidráulica.

A adição de surfactante ao sistema estudado mostrou-se necessária para a obtenção de uma suspensão homogênea, promovendo o aumento dos níveis de fluidez e decréscimo dos níveis de torque durante o ciclo de cisalhamento.

Os resultados apresentados mostram-se muito promissores com relação à otimização de forma sistemática da dispersão e das propriedades reológicas de concretos refratários no sistema $\mathrm{Al}_{2} \mathrm{O}_{3}-\mathrm{SiC}-\mathrm{SiO}_{2}-\mathrm{C}$.

\section{AGRADECIMENTOS}

À Alcoa Alumínio S.A. e à Unimetal pelo fornecimento das matérias-primas e à FAPESP pelo apoio financeiro recebido.

\section{REFERÊNCIAS}

[1] A. R. Studart, W. Zhong, V. C. Pandolfelli, "Rheological design of zero-cement self-flow castables", Am. Ceram. Soc. Bull. 78, 5 (1999) 65-72.

[2] I. R. Oliveira, A. R. Studart, V. C. Pandolfelli, B. A. Menegazzo, "Zero-cement refractory castables", Am. Ceram. Soc. Bull. 81, 12 (2002) 27-34.

[3] A. R. Studart, V. C. Pandolfelli, "Thermomechanical behavior of zero-cement high-alumina castables", Am. Ceram. Soc. Bull. 79, 10 (2000) 53-60.

[4] R. G. Pileggi, V. C. Pandolfelli, A. E. Paiva, J. Gallo, "Novel rheometer for refractory castables", Am. Ceram. Soc. Bull. 79, 1 (2000) 54-58.

[5] R. G. Pileggi, A. R. Studart, V. C. Pandolfelli, "How mixing affects the rheology of refractory castables", Am. Ceram. Soc. Bull. Part I- 80, 6 (2001) 27-31; Part II- 80, 7 (2001) 38-42. [6] I. R. Oliveira, A. R. Studart, F. A. Silva Júnior, V. C. Pandolfelli, "Estabilização de suspensões aquosas contendo grafite", Cerâmica 46, 300 (2000) 186-195.

[7] K. M. Parker, J. H. Sharp, "Refractory calcium aluminate cements", Brit. Ceram. Trans. J. 81, 2 (1982) 35-42.

[8] I. R. Oliveira, A. R. Studart, F. A. O. Valenzuela, V. C. Pandolfelli, "Influence of additives on the working time of the ultra-low cement content refractory castable", Am. Ceram. Soc. Bull. (2002) submetido.

(Rec. 06/05/02, Rev. 02/07/02, Ac. 21/07/02) 